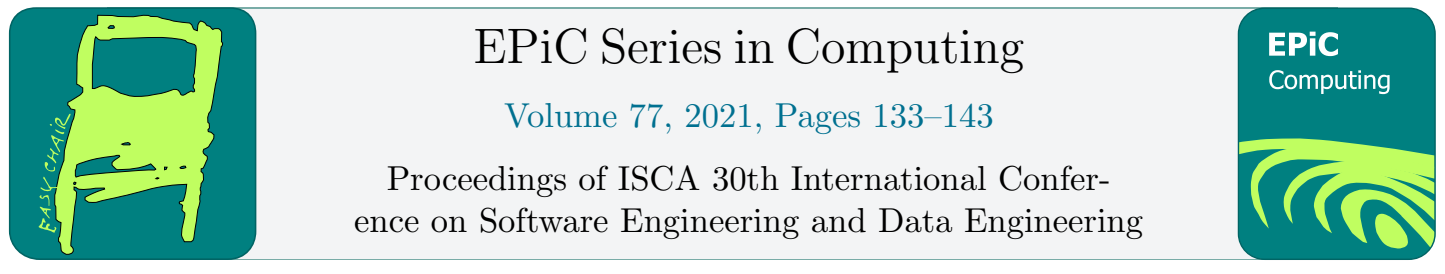

\title{
Studying the COVID-19 Impact on the Antarctic Glacier Melting Rate
}

\author{
Mason Chen ${ }^{1 *}$, and Charles Chen ${ }^{2}$ \\ ${ }^{1}$ Stanford University OHS, Redwood City, CA, USA \\ ${ }^{2}$ STEAMS, San Jose, CA, USA \\ mason05@ohs.stanford.edu, charles.chen.training@gmail.com
}

\begin{abstract}
This paper will study the Time Series Antarctic Glacier Mass from April 2002 to March 2021. The objective of this paper is to forecast the Antarctic Glacier Mass level for 2021 to 2041. The Science studied is the Geoscience of the Glacier; the Technology applied is the GRACE-FO satellites to collect the Glacier Ice Sheet Mass data; Engineering focuses on the COVID-19 impact on the Glacier melting rate; and mathematical/statistical tools like Time Series ARIMA models are applied. Although the Glacier melting rate sped up recently before 2020, the COVID-19 situation might have slowed down the rate of glacier melting in 2020 in both Antarctic and Greenland. During 2020 COVID-19 period, Antarctic Glacier Mass seasonal pattern became a smoother single-peak cyclic pattern which is different from the double-peak cyclic pattern in 2002 to 2019. Authors conducted both non-seasonal and seasonal ARIMA models and concluded that only the Seasonal ARIMA Forecasting modeling algorithm can detect more reliable insights of the relatively small pattern change during 2020 period. The COVID-19 factor might have made certain impact on the Antarctic Glacier Melting rate. The Glacier Melting rate may have been slowed down by $20 \%$ in the 2020-2021 period.
\end{abstract}

\section{Introduction}

This project would study the Antarctic Glacier Mass data from 2002-2021 March. The objective is to use the Time Series platform to examine the time series Glacier data to predict the Glacier crisis for the next twenty years (2021-2041).

\subsection{Scientific Research Literature and Technology: GRACE-FO}

The global climate has been spiraling out of control due to the Global Warming effect (Arnold 2011). The Gravity Recovery and Climate Experiment Follow-On (GRACE-FO) mission is a 
partnership between NASA and the German Research Centre for Geosciences (GFZ). GRACE-FO aims to test a new technology designed to dramatically improve the already remarkable precision of its measurement system. GRACE-FO is a successor to the original GRACE mission, which orbited Earth from 2002-2017. Global surface mass anomalies are observed by the GRACE-FO satellites. Over land, red colors indicate below-average terrestrial water amounts, while blue colors show above-average water amounts (including ice, snow, soil moisture and groundwater). Over oceans, red colors indicate below-average ocean pressure, while blue colors show above-average pressure. Ocean pressure changes are related to large-scale ocean current variations, as well as overall sea level changes from ocean mass changes.

\subsection{Engineering: Antarctic Glacier Melting Crisis}

An Antarctic glacier larger than the UK is at risk of breaking up after scientists discovered more warm water flowing underneath it than previously thought. Over the past few years, teams of scientists have been crisscrossing the remote and inaccessible region on Antarctica's western edge to try to understand how fast the ice is melting and what the consequences for the rest of the world might be. "What happens in west Antarctica is of great societal importance," said Dr Robert Larter [1], a scientist with the British Antarctic Survey and principal investigator with the International Thwaites Glacier Collaboration. Glacier melting is the biggest factor in future sea level rise [2].

\subsection{Mathematics: Time Series and Forecast}

Time Series Analysis and Forecasting modeling were utilized on the GRACE-FO Glacier Mass data. Climatology research has used Time Series and Forecasting model such as ARIMA to forecast the weather temperature and study the global warming trend [3]. In this paper, the Time Series Decomposition and Smoothing Models will be compared on their Forecasting Capabilities for the next 20 years (2021-2041).

\section{Data Collection and Sampling Plan}

The data source for this paper is from the NASA GRACE-FO satellites' data of the Antarctic Ice Sheet Mass Trend as shown in Figure 1 which collects monthly averages of the images collected from satellites. 


\section{ANTARCTICA MASS VARIATION SINCE 2002}

Data source: Ice mass measurement by NASA's GRACE satellites. Gap represents time between missions.

Credit: NASA

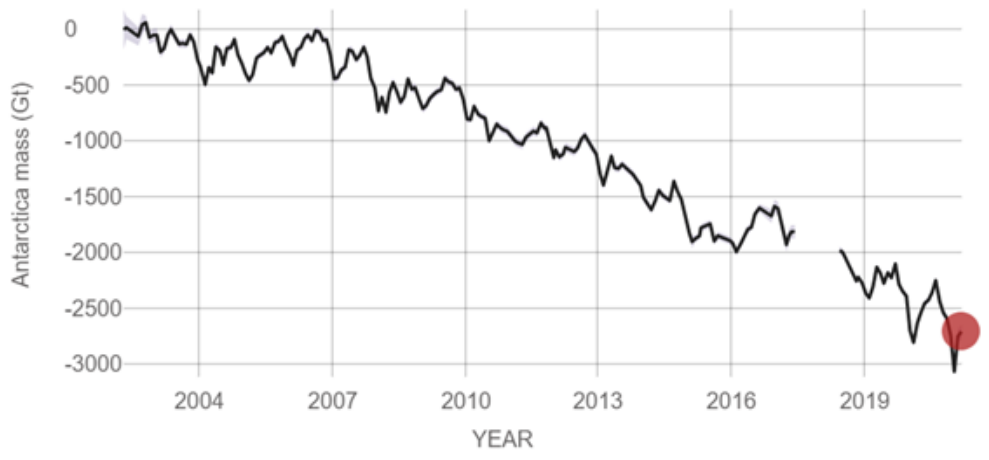

Click+drag

to zoom
RATE OF CHANGE

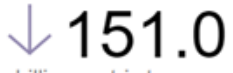

billion metric tons per

year
Get Data: HTTP | Snapshot: PNG

Figure 1: Antarctic Monthly Mass Trend

The Glacier Mass raw data was uploaded to the JMP platform from the NASA GRACE-FO website as shown in Figure 2.

\begin{tabular}{|c|c|c|c|c|c|c|c|}
\hline \multirow[t]{7}{*}{$D$ Source } & $\begin{array}{ll}\triangleleft & \text { V } \\
- & \text { E }\end{array}$ & $\begin{array}{l}\text { Antarctic mass } \\
\text { (Gigatonnes) }\end{array}$ & Year & Month & Year-Month & $\begin{array}{c}\text { Antarctic mass (Gigatonnes) } \\
\text { (Detrended) }\end{array}$ & $\begin{array}{l}\text { Antarctic mass (Gigatonnes) } \\
\text { (Remove } 12 \text { unit cycle) }\end{array}$ \\
\hline & 1 & 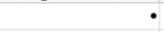 & 2002 & 1 & $01 / 2002$ & - & - \\
\hline & 2 & - & 2002 & 2 & $02 / 2002$ & • & - \\
\hline & 3 & - & 2002 & 3 & $03 / 2002$ & • & • \\
\hline & 4 & 0 & 2002 & 4 & $04 / 2002$ & -312.2540246 & 141.67839272 \\
\hline & 5 & 18.36 & 2002 & 5 & $05 / 2002$ & -281.1661726 & 51.559262294 \\
\hline & 6 & - & 2002 & 6 & $06 / 2002$ & • & - \\
\hline \multirow{2}{*}{ - Columns (6/0) } & 7 & - & 2002 & 7 & $07 / 2002$ & • & - \\
\hline & 8 & -59.82 & 2002 & 8 & $08 / 2002$ & -321.1626167 & -285.6739764 \\
\hline a & 9 & 45.54 & 2002 & 9 & $09 / 2002$ & -203.0747647 & -166.6549122 \\
\hline \multirow{10}{*}{$\begin{array}{l}4 \text { Antarcti...tonnes) } \\
4 \text { Year } \\
4 \text { Month } \\
4 \text { Year-Month } \frac{\text { औै }}{4} \\
\text { Antarcti...rended) } \\
4 \text { Antarcti...it cycle) }\end{array}$} & 10 & 62.69 & 2002 & 10 & $10 / 2002$ & -173.1969127 & -78.98839272 \\
\hline & 11 & -69.03 & 2002 & 11 & $11 / 2002$ & -292.1890607 & -102.2292623 \\
\hline & 12 & -49.78 & 2002 & 12 & $12 / 2002$ & -260.2112087 & 34.395583658 \\
\hline & 13 & -48.71 & 2003 & 1 & $01 / 2003$ & -246.4133567 & 130.28564995 \\
\hline & 14 & -200.03 & 2003 & 2 & $02 / 2003$ & -385.0055048 & 25.823976382 \\
\hline & 15 & -171.49 & 2003 & 3 & $03 / 2003$ & -343.7376528 & 40.70491224 \\
\hline & 16 & -43.66 & 2003 & 4 & $04 / 2003$ & -203.1798008 & 98.018392724 \\
\hline & 17 & 0.79 & 2003 & 5 & $05 / 2003$ & -146.0019488 & 33.989262294 \\
\hline & 18 & - & 2003 & 6 & $06 / 2003$ & - & - \\
\hline & 19 & -128.94 & 2003 & 7 & $07 / 2003$ & -250.2762448 & -307.9356499 \\
\hline Rows & 20 & -122.41 & 2003 & 8 & $08 / 2003$ & -231.0183929 & -348.2639764 \\
\hline \multirow{4}{*}{$\begin{array}{l}\text { All rows } \\
\text { Selected } \\
\text { Excluded } \\
\text { Hidden } \\
\text { Labeled }\end{array}$} & 21 & -130.92 & 2003 & 9 & 09/2003 & -226.8005409 & -343.1149122 \\
\hline & 22 & -48.06 & 2003 & 10 & $10 / 2003$ & -131.2126889 & -189.7383927 \\
\hline & 23 & -107.58 & 2003 & 11 & $11 / 2003$ & -178.0048369 & -140.7792623 \\
\hline & 24 & -273.11 & 2003 & 12 & $12 / 2003$ & -330.8069849 & -188.9344163 \\
\hline
\end{tabular}

Figure 2: Glacier Mass Monthly Row Data File 


\section{Time Series Basic Analysis}

Conduct JMP 16 Time Series and Forecasting Platforms on the Glacier Mass data. The main objective in this Section: any change in Antarctic glacier melting rate during COVID-19 period in 20202021 ?

\subsection{Control Chart Analysis}

To visualize Antarctic Glacier Mass trend from 2002-2021, JMP Control Chart Builder platform was used. In Figure 3, the Glacier Mass data was plotted in Individual Control Chart format [4-7]. Y Axis is the Antarctic Mass data and $\mathrm{X}$ axis is the Month/Year time domain. Y axis scale was set zero at the 2002 April. The Mass data reported was compared relatively to the 2002 April data in Gigatons (GT). The downward trending pattern was observed since 2002 and the downward slope was getting steeper after 2007. An interesting finding is that the Glacier Mass melting rate was slowed down in Antarctic in 2020. This observation may be related to COVID-19 factor. Authors have also found a similar trending observation happened in Greenland in 2020. Between September 2018 and August 2019, the Greenland Ice Sheet set a record for ice loss (532 plus or minus 58 billion metric tons). Between September 2019 and August 2020, the rate of ice loss from the Greenland Ice Sheet was much lower (293 plus or minus 66 billion metric tons), but still above the 2002-2020 average measured by GRACE. Average ice loss for Greenland over the full 18-year record was 268 plus or minus 14 billion metric tons per year. This slow down observation may be due to less Human activity and air pollution globally during COVID-19 pandemic period. Authors have been monitoring this COVID-19 factor and may share more findings in next Antarctic Glacier Mass Time Series publication.

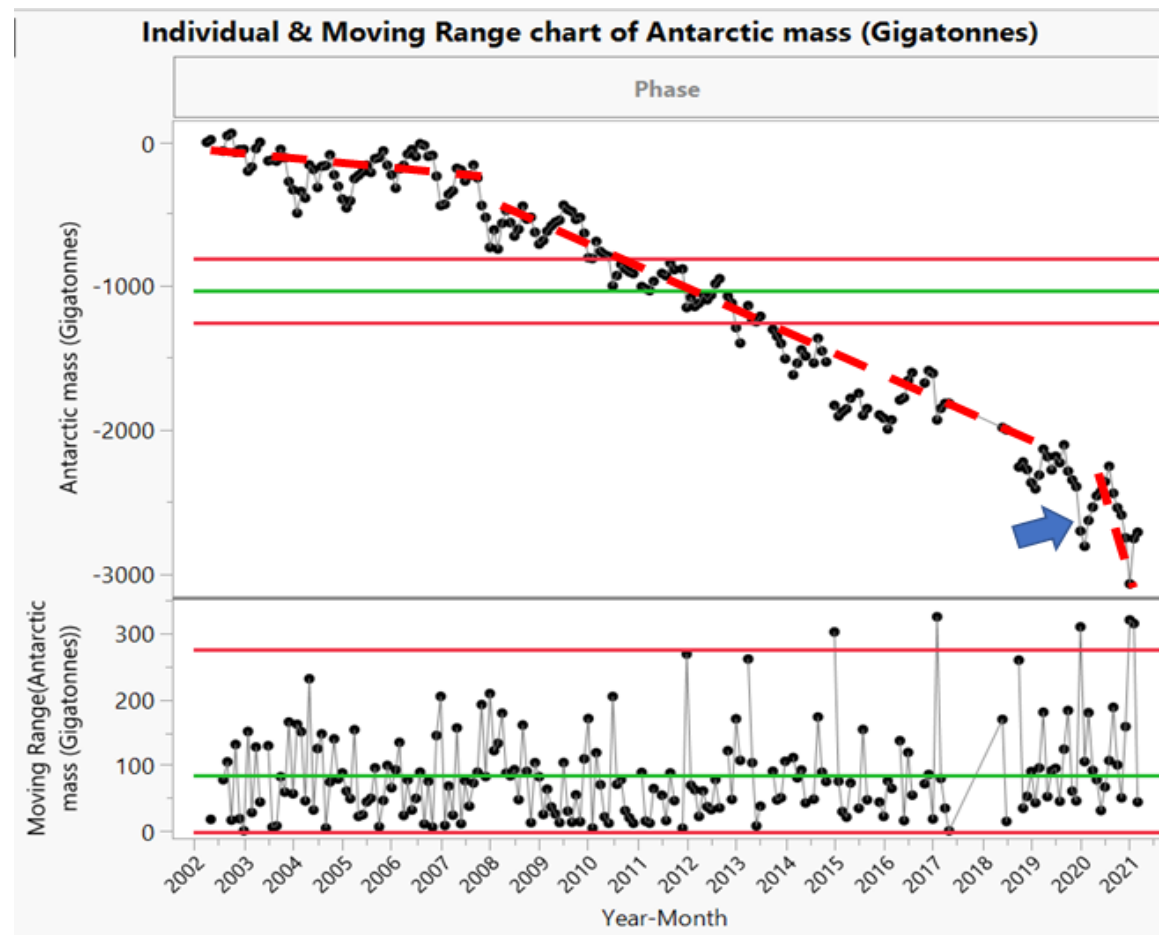

Figure 3: Antarctic JMP Glacier Mass Control Chart Builder Analysis 


\subsection{Study 2020 Antarctic Glacier Mass Trend}

Historical Monthly Antarctic Glacier Mass records were summarized in Figure 4. The lowest points were in the Jan.-Mar. (their warm months). The highest points were in the August to October (their cold months). Interesting portion is their April to July months. There seems another small peak-valley cycle happened in April to July.

\begin{tabular}{|r|r|r|}
\hline & \multicolumn{2}{|c|}{ Antarctic mass (Gigatonnes) } \\
\hline Month & Mean & Std Dev \\
\hline 1 & -1242 & 907 \\
2 & -1252 & 880 \\
3 & -1212 & 872 \\
4 & -956 & 781 \\
5 & -954 & 806 \\
6 & -1059 & 822 \\
7 & -1012 & 775 \\
8 & -889 & 789 \\
9 & -790 & 807 \\
10 & -880 & 894 \\
11 & -982 & 874 \\
12 & -1060 & 852 \\
\hline
\end{tabular}

Figure 4: Summary of Historical Antarctic Glacier Mass Data in 2002-2021

To investigate this double-peak mode, data was rearranged in histogram analysis across 2002-2021 years as shown in Figure 5. Clearly, the Histogram shapes were quite different: double peaks patterns observed before 2020 and single peaks pattern observed in 2020 COVID-19 period.
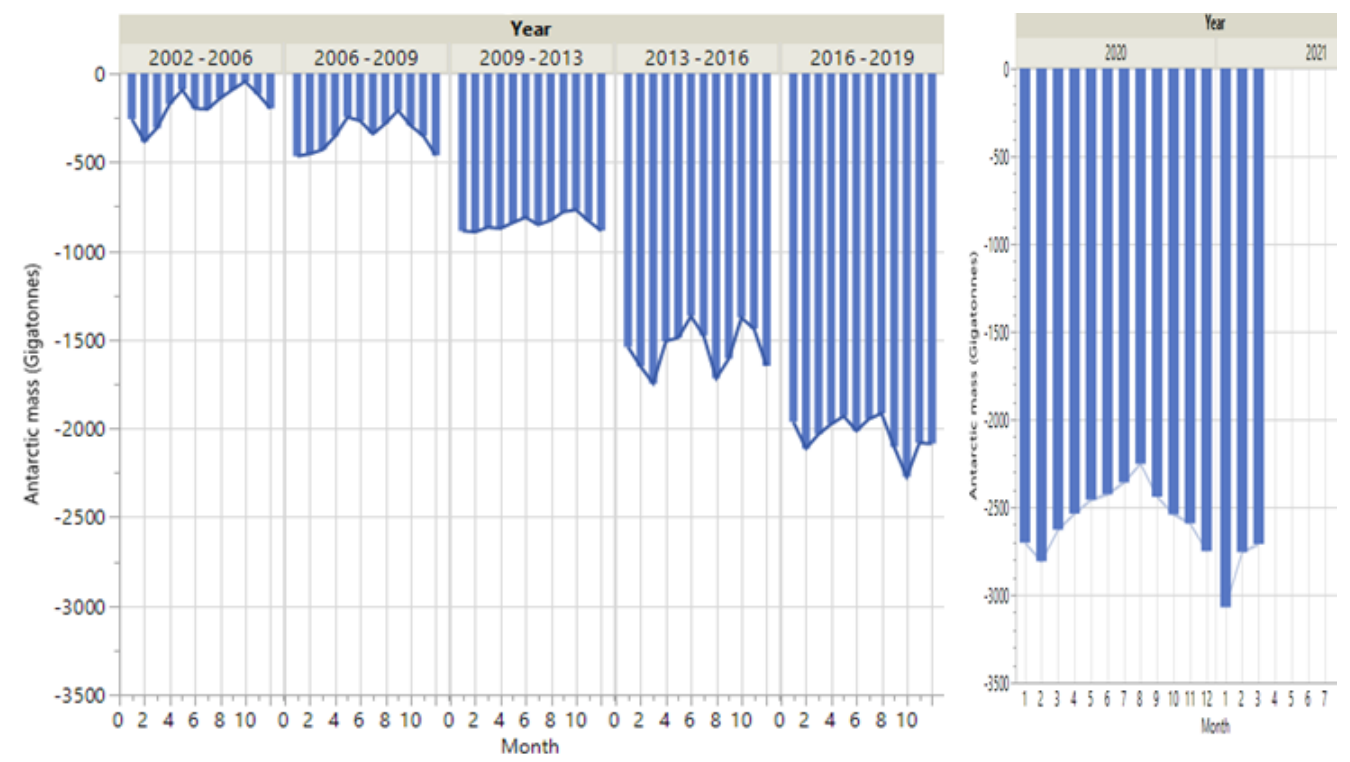

Figure 5: Histogram Analysis of the Historical Antarctic Glacier Mass Data 
To further investigate the patterns, year 2019 and year 2020 Antarctic Glacier Mass data were plotted in the Time Series Plot together with 2002-2021 Regression Fit Model and 95\% Confidence and Prediction Intervals as shown in Figure 6. During COVID-19 year 2020 period, the Antarctic Glacier Mass pattern was much smoother as a single-peak mountain. Less Human activity during the lock down season may transform the Glacier ecology back to the Natural mode which has reflected a natural seasonal pattern of the Glacier Melting mechanism. Year 2019 Glacier Pattern was a typical curve for 2002-2018. The curve was not in a smooth seasonal pattern from March to July for certain special factors. Somehow, year 2020 could avoid those special factors. At this moment, there is no direct evidence or correlation study between those unknown special factors and COVID-19.

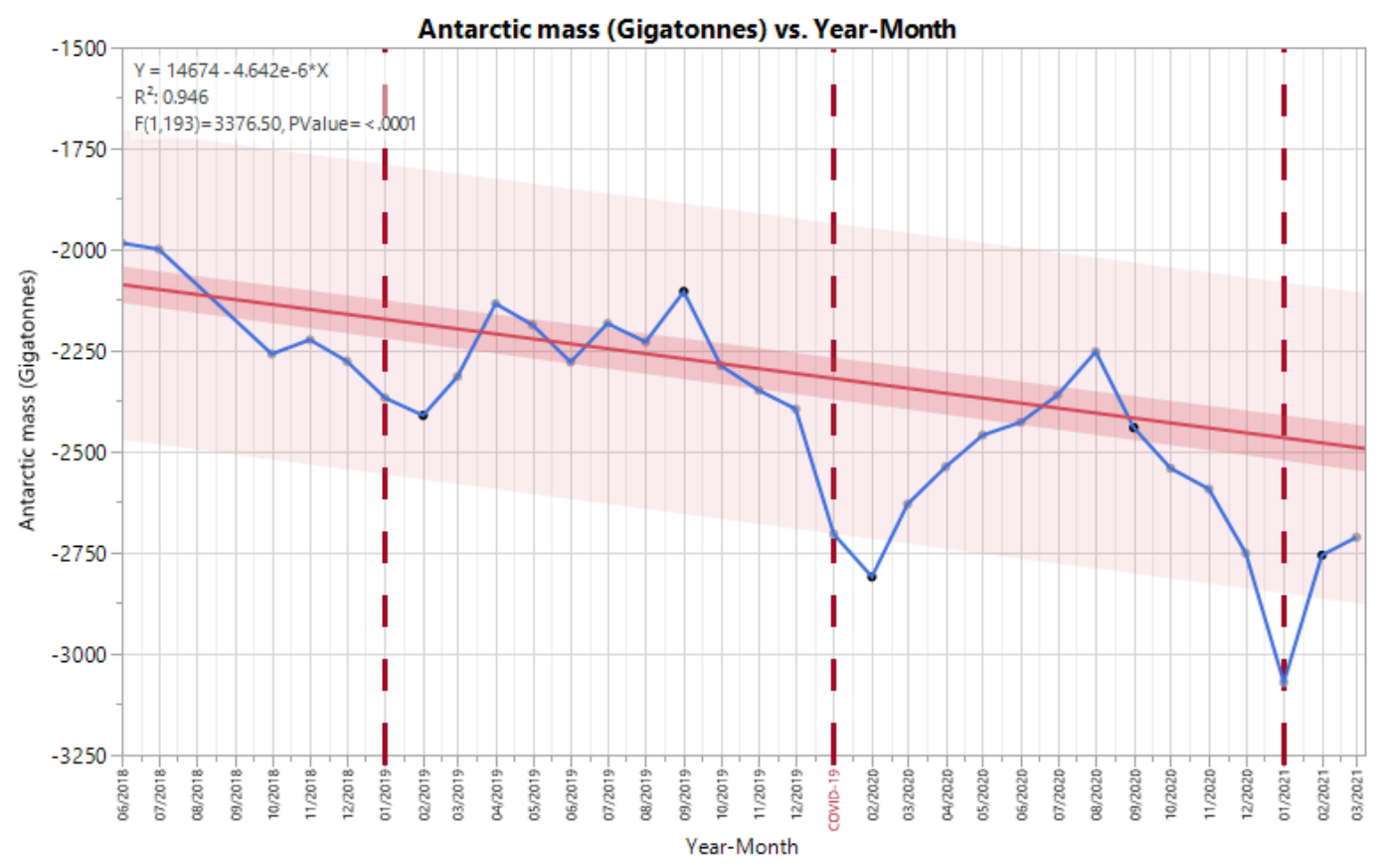

Figure 6: Compare Antarctic Glacier Mass data between year 2019 and year 2020

\section{Time Series ARIMA Model}

To further study the COVID-19 factor, authors would conduct the Time Series ARIMA model w/wo including the 2020 Jan.-2021 Mar. data for direct model comparison.

\subsection{Non-Seasonal Time Series Analysis}

ARIMA has three mathematical components [8]: Autoregression (AR), Integration (I) and Moving Average (MA). Autoregression (AR): refers to a model that shows a changing variable that regresses on its own lagged, or prior, values. Integrated (I): represents the differencing of raw observations to allow for the time series to become stationary, i.e., data values are replaced by the difference between the data values and the previous values. Moving average (MA): incorporates the dependency between an observation and a residual error from a moving average model applied to lagged observations. There are two ARIMA types: one is Non-Seasonal and one is Seasonal. The difference is whether there is a fixed Seasonal component observed or detected in the Time Series Data. A Non-Seasonal ARIMA 
model is commonly denoted ARIMA(p,d,q). The AR "p" number considers the Autoregression AR module by integrating the historical values in exponentially decaying algorithm. The I " $\mathrm{d}$ " number considers the Integration Differencing I module by differencing the data points to detect the trend component. The MA "q" number considers the Moving Average MA module by smoothing the error term exponentially. If any of $\mathrm{p}, \mathrm{d}$, or $\mathrm{q}$ are zero, the corresponding letters are often dropped. For example, if $\mathrm{p}$ and $\mathrm{d}$ are zero, then the model would simply be a moving average model, denoted as MA(q). The Seasonal ARIMA model would be addressed in Section 4.2. As shown in Figure 7, NonSeasonal ARIMA model was conducted w/wo including the COVID-19 period data (Jan.2020- Mar. 2021). The top two Non-Seasonal Models based on the AIC criteria $[9,10]$ are identical between these two cases. Regarding the model goodness of fit, R-Square are similar between two datasets. Due to 15 DF difference, hard to tell which dataset has better ARIMA fit on the other selection criteria. In general, from the non-seasonal ARIMA modeling, we did not detect any significant COVID-19 factor

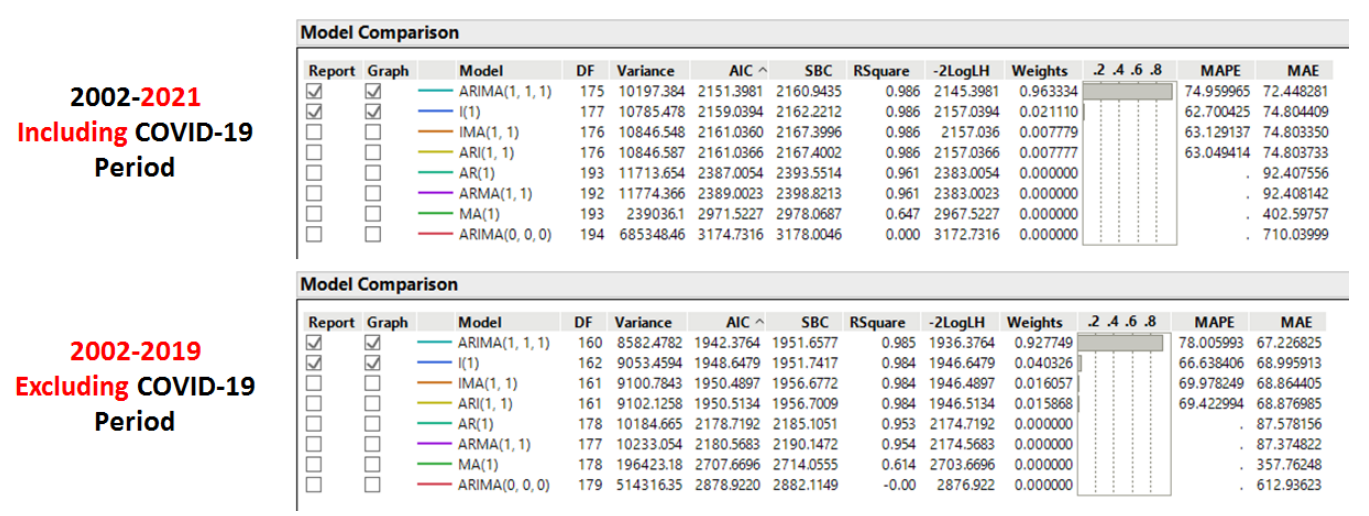

Figure 7: Non-Seasonal ARIMA Model Analysis

Authors are particularly interested in comparing the ARIMA $(0,1,0)$ model between w/wo COVID19 data analysis as shown in Figure 8. ARIMA $(0,1,0)$ model may indicate that the second component $\mathrm{I}=1=>$ integrating or differencing (Linear Trend). Parameter estimate $\mathrm{t}$ test $\mathrm{P}$-Value $>0.05=>$ intercept (constant) $\mathrm{c}$ is not significant even though a clear downward trend term observed. The downward trend term may be masked by the seasonal component with non-seasonal ARIMA model. P-Values for Parameter Estimate are similar between two datasets which may imply that little No COVID-19 factor detected. The parameter estimates analysis has estimated Intercept $=-10.43$ and -9.44 (downward trend slope). Though, the t test P-value $=0.18>0.05$ (could not reject the Null Hypothesis of Intercept=0). There are two possible reasons of not rejecting the Null Hypothesis: (1) there is a strong seasonal component existing in the Glacier Mass data. In the Non-Seasonal ARIMA model, this strong Seasonal component signal would be treated as Non-Seasonal Noise and weaken the Signal-Noise Ratio in Parameter Estimate $t$ test, and (2) the sample size may not be sufficient. Glacier data was collected in 2002-2021 (20 years). If the seasonal component is very strong (12 months), then 20 years of sample size (signal) may not be sufficient as compared to 12 months Seasonal (Noise) in the Non-Seasonal ARIMA model. Also, the downward slope is about $10 \%$ less steeper if excluded the most recent 20202021 COVID data. This finding may be against our Hypothesis that the Antarctic Glacier's melting rate has been slowed down during 2020-2021 COVID season. Let's wait and see what Seasonal ARIMA model may find out the same or different regarding the COVID-19 factor. 
Studying COVID-19 Impact on the Antarctic Glacier Melting Rate Mason Chen and Charles Chen
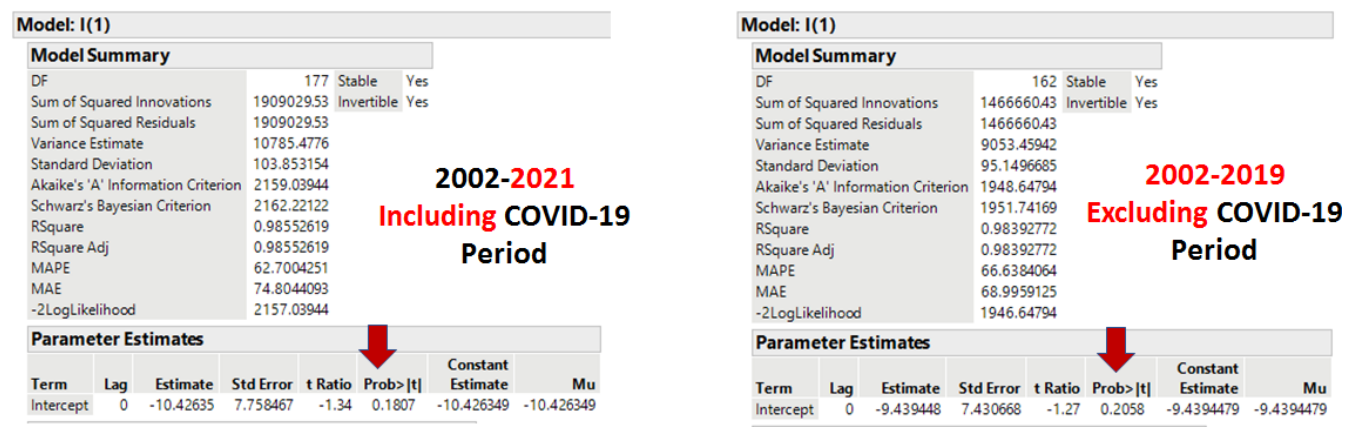

Figure 8: Non-Seasonal ARIMA Model and Parameter Estimate w/wo COVID-19 period

\subsection{Seasonal Time Series Analysis}

In addition to Non-Seasonal ARIMA model, the Seasonal ARIMA model has added the Seasonal Component as $(\mathrm{p}, \mathrm{d}, \mathrm{q})(\mathrm{P}, \mathrm{D}, \mathrm{Q}) \mathrm{m}$. (P, D, Q) is based on the Seasonal pattern. $\mathrm{m}=12$ here is representing 12 months in a season (year). For example, in previous ARIMA $(1,1,1)$ model, " $d=1$ " means the trend component $\mathrm{d}=1$ is a straight line in Forecasting. " $\mathrm{d}=1$ " is the differencing (delta) is constant between any two consecutive months, resulting in a constant slope of linear trend. In Seasonal ARIMA model, the " $\mathrm{D}=1$ " component would compare the same month of two consecutive years (season = one year). This " $\mathrm{D}=1$ " component in the Seasonal ARIMA model could detect any year to year non-linear longterm trend in addition to non-Seasonal " $d=1$ " linear trend model. To simply the Seasonal ARIMA model list for model comparison, the Non-Seasonal ARIMA portion has been limited to I(1). Both the AR and MA modules would be addressed in Seasonal portion (P,D,Q) better. Non-Seasonal I (1) was kept in the Seasonal ARIMA model because it may make more sense to consider both the local linear trend of differencing between two consecutive months and the global non-linear trend of differencing between two consecutive years based on 12 months of a Season. Through these model selection list, we may directly compare the strength of the short term trend Vs. the long term trend through the Seasonal ARIMA Model analysis. The relative strength of these two trend methods may indicate whether the Antarctic Glacier may melt faster in the next 20 years. As shown in Figure 9, the top Seasonal ARIMA models are ARIMA $(0,1,0)(0,1,1) 12$ same for w/wo COVID period. Seasonal ARIMA models were ranked based on AIC criteria as shown in Figure 9. ARIMA $(0,1,0)(0,1,1) 12$ was identified as the best model. Previous non-Seasonal I (1) was on the bottom. This new Seasonal ARIMA model may indicate four major findings: (1) Seasonal component is very strong in Antarctic Glacier Melting forecasting, (2) Non-Linear long term trend " $\mathrm{D}=1$ " is significant, (3) Autoregression can be ignored in ARIMA, and (4) Moving Average method is necessary in ARIMA.

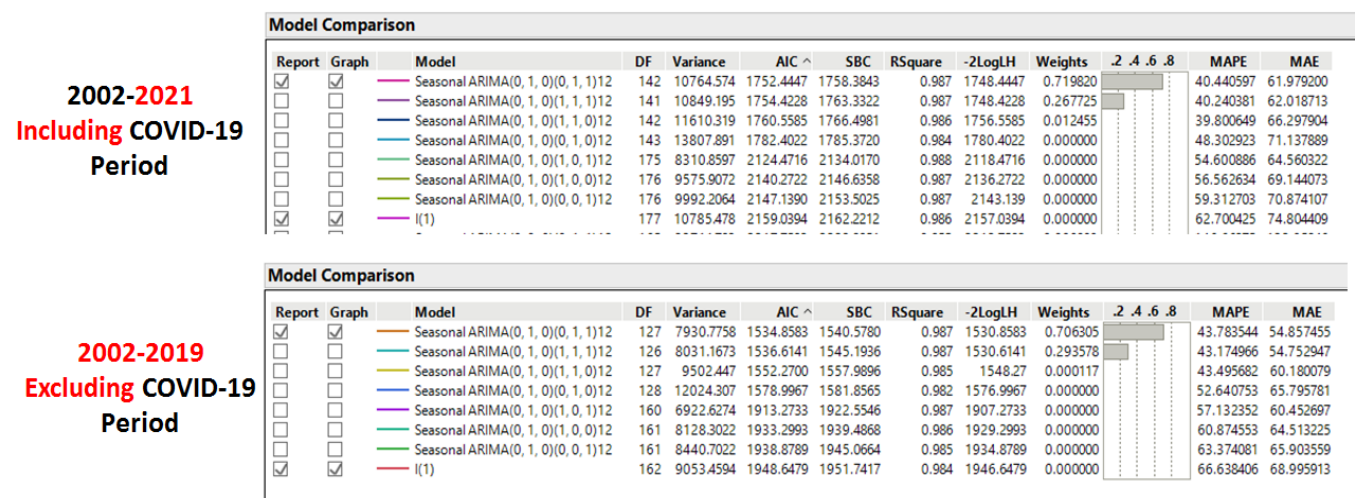

Figure 9: Remove Seasonal Component Analysis 
In Figure 10 Seasonal ARIMA Model Summary and Parameter Estimates, the MA module $t$ test was significant $(\mathrm{P}$-value $<0.05)$ and the Intercept Trend component was relatively weaker $(\mathrm{P}$-Value $>$ 0.05). The significant MA term may indicate the importance of the smoothing out the random error noise for forecasting in the Seasonal ARIMA model [9]. Even the nonlinear long term intercept is not significant, the yearly decaying slope is still $-2.38 \mathrm{GT} / \mathrm{year}$ as compared to $-10.43 \mathrm{GT} / \mathrm{month}$ or -125 GT/year. Even with less than $2 \%$ contribution of this non-linear trend term, after 10 years, the contribution or impact of the Forecasting accuracy will be near $20 \%$ (faster Glacier melting rate than the non-seasonal forecasting). Therefore, the Seasonal ARIMA model has significantly upgraded the forecasting power of the long-term Glacier Forecasting. If excluding the COVID-19 period, the yearly decaying slope is steeper at -2.91 GT/year. The COVID-19 period has slowed down the long-term yearly Glacier melting rate which is not consistent with the previous Non-seasonal ARIMA model. The possible reason is that the COVID-19 period has a different seasonal pattern in 2020 which has helped removed or mitigated the random noise level, resulting in a stronger seasonal component and trend component.
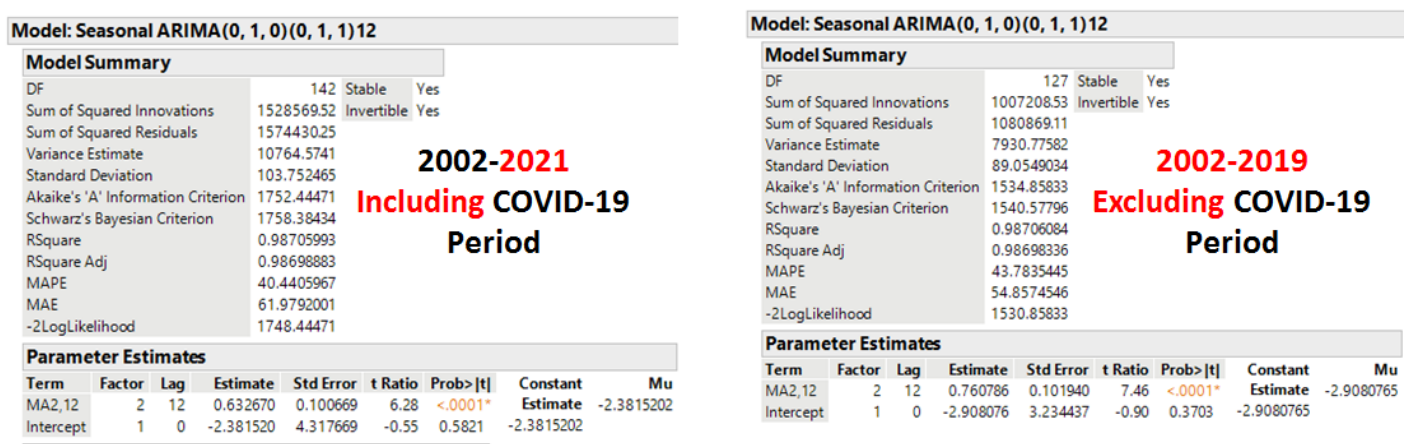

Figure 10: Seasonal ARIMA Model Summary and Parameter Estimates

\subsection{Seasonal Time Series Forecasting}

Seasonal ARIMA model [10-12] has significantly upgraded the forecasting power of the long-term Glacier Forecasting. As shown in the Figure 11, both non-seasonal and seasonal ARIMA forecasting were side by side compared. On the left hand chart, including COVID-19 period, the Seasonal ARIMA model has shown the seasonal pattern and faster decaying trend than the non-Seasonal model. After excluded the COVID-19 period data, there is less difference in Forecasting between the non-seasonal ARIMA trend slope and the seasonal ARIMA trend slope This new observation may be related to our previous "two-peak" Vs, "one-peak" seasonal pattern. We may consider this hypothesis: during the COVID-19 period, the seasonal pattern recovered to its natural one-peak pattern and enhance the 12 months-seasonal component. Therefore, in the left chart including the COVID-19 period, the trend component is less masked by the seasonal component, resulting in a stronger trend component in the seasonal ARIMA. On the right hand chart, excluding COVID-19 period, there is much smaller trend forecasting difference between the Non-Seasonal and Seasonal ARIMA model. The seasonal ARIMA forecasting has less power to improve the long-term yearly trend component. In general, the 2020 COVID period has not just changed the seasonal month-month pattern but also improved the detection capability of the long-term yearly year-year decaying trend. 

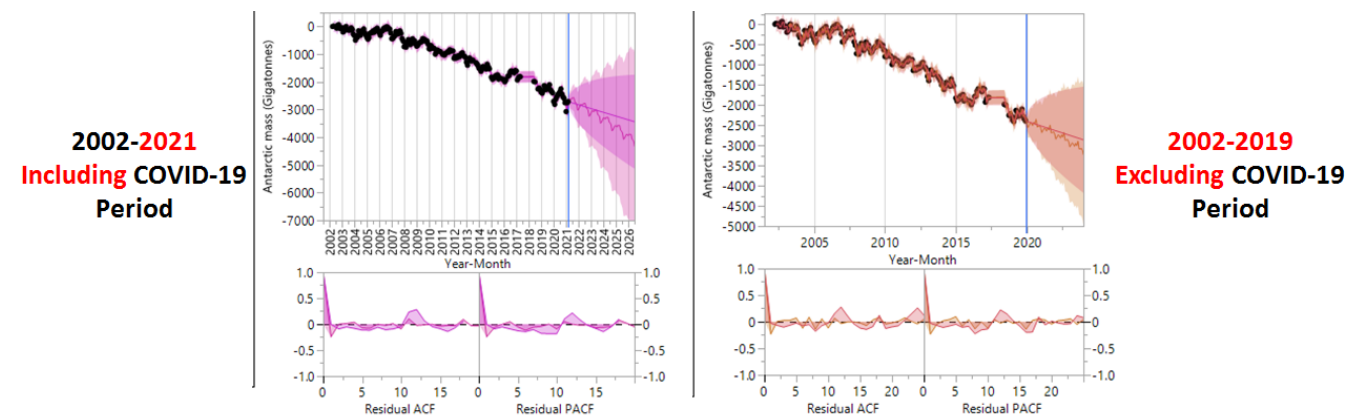

Figure 11: Seasonal ARIMA Forecasting w/wo COVID Period

\subsection{Validate COVID-19 Factor on Antarctic Glacier Melting}

To investigate the COVID-19 factor on comparing the Seasonal ARIMA Forecasting results as shown in Figure 12, the Blue Curve is the actual data from 2020 Jan.-2021 Mar. Glacier Mass Data collected and the Red curve is the forecasted data based on the Seasonal ARIMA model built based on the data from 2002 Apr.to 2019 Dec. If Blue and Red lines are overlapping each other, the COVID period data has little impact on the Forecasting for 2021-2041. During the COVID plotted period, there is smaller deviations during the Antarctic's colder months but larger deviations in warmer months. This observation may indicate that the Antarctic Glacier Mass pattern is different during 2020 COVID period. Though, with limited Glacier Mass sample data and sample size, we may not draw meaningful conclusions. Can consider to add other critical information such as Temp, Humidity, Air Pollution data.

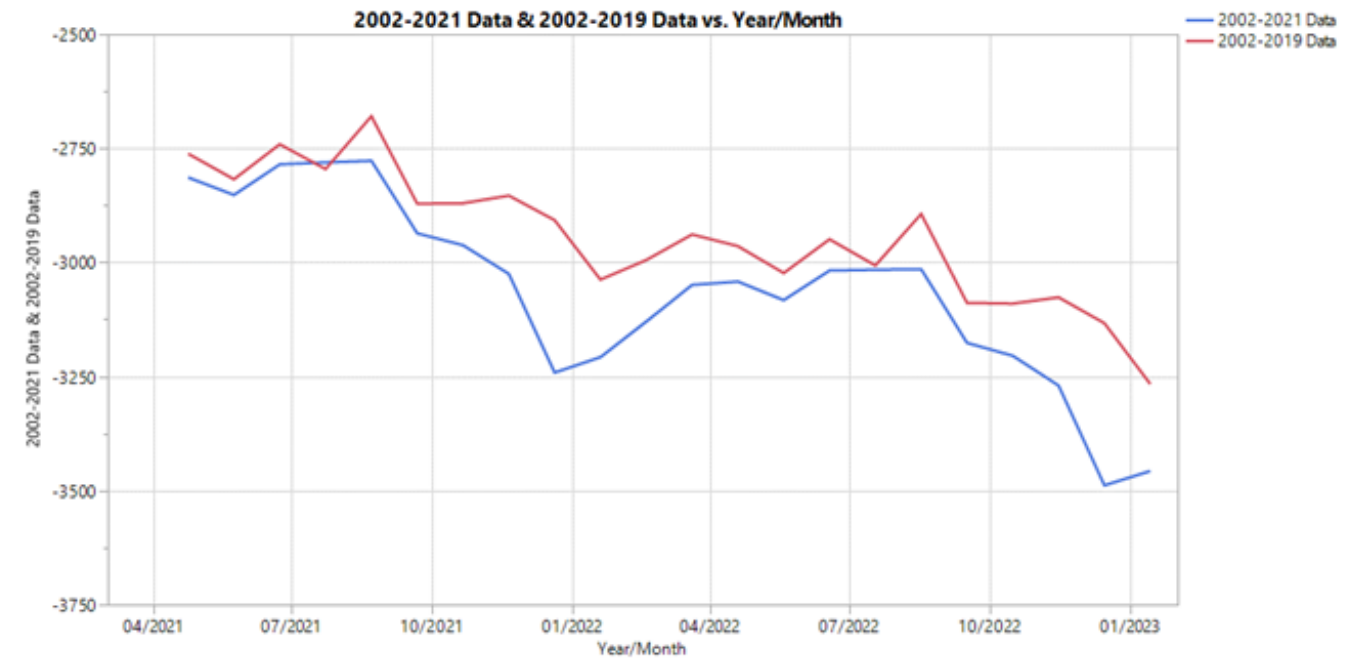

Figure 12: Validate COVID-19 period factor in Seasonal ARIMA model

\section{Conclusions}

The COVID-19 impact on the Antarctic Glacier Melting was conducted through the Time Series ARIMA Model. Although a significant deviation on both the Non-Seasonal ARIMA and Seasonal ARIMA modeling was not observed, the "1-Peak" Seasonal Pattern in 2000 differed substantially from the "2-Peaks" Seasonal Pattern from 2002 to 2019. A stronger Forecasting "Trend" component in the 
Studying COVID-19 Impact on the Antarctic Glacier Melting Rate Mason Chen and Charles Chen

Seasonal ARIMA model was observed as compared to the non-Seasonal ARIMA model. Nevertheless, due to the limited Glacier Mass sample data and sample size, other critical information such as temperature, humidity, and air pollution data may be needed to draw more meaningful conclusions.

\section{References}

[1] D. Arnold, "The Ethics of Global Climate Change", New York: Cambridge University Press. doi:10.1017/CBO9780511732294, 2011

[2] N. Bindoff and P. Stott, "Detection and Attribution of Climate Change: from Global to Regional", in Stocker. 867-952, 2013

[3] R. Baillie and SK. Chung, "Modeling and forecasting from trend-stationary long memory models with applications to climatology", International Journal of Forecasting. Volume 18, Issue 2, Pages 215-226, 2002

[4] AIAG, "Statistical Process Control". 2nd ed. Troy, MI: Automotive Industry Action Group, 2005

[5] L. Nelson, "The Shewhart Control Chart-Tests for Special Causes." Journal of Quality Technology 15:237-239, 1984

[6] L. Nelson, "Interpreting Shewhart X Control Charts." Journal of Quality Technology 17:114116,1985

[7] D.J. Wheeler, "Advanced Topics in Statistical Process Control”. 2nd ed. Knoxville, TN: SPC Press, 2004

[8] G.E. Box, G.M. Jenkins, and G.C. Reinsel, "Time Series Analysis: Forecasting and Control". 3rd ed. Englewood Cliffs, NJ: Prentice-Hall, 1994.

[9] J. Shiskin, A.H. Young, and J.C. Musgrave, "The X-11 Variant of the Census Method II Seasonal Adjustment Program. Technical Report 15, US Department of Commerce, Bureau of the Census", 1967

[10] K.P. Burnham, D.R. Andersen, and K.P. Huyvaert, "AIC Model Selection and Multimodel Inference in Behavioral Ecology: Some Background, Observations, and Comparisons." Behavioral Ecology and Sociobiology 65:23-35, 2011

[11] R.J. Hyndman et. al, "Forecasting with Exponential Smoothing: The State Space Approach". Berlin: Springer-Verlag, 2008

[12] K.P. Burnham and D.R. Anderson, "Multimodel Inference: Understanding AIC and BIC in Model Selection.”, Sociological Methods and Research 33:261-304, 2004 